IN-SITU DETERMINATION OF POLYMORPHIC PHASE DIAGRAMS E. Aret ${ }^{1}$ H. Meekes ${ }^{1}$ E. Vlieg ${ }^{1}$ G. Deroover ${ }^{2}$

${ }^{1}$ University of Nijmegen NSRIM Laboratory for Solid State Chemistry Toernooiveld 1 NIJMEGEN 6525 ED NETHERLANDS ${ }^{2}$ Agfa-Gevaert N.V., Septestraat 27, B-2640, Mortsel, Belgium

One of the key issues in the understanding of polymorphic phase behavior is the effect of kinetics versus thermodynamics on the nucleation and growth. The effect of kinetics is often studied by nucleation experiments in vessels, involving the simultaneous formation of many crystals.

An important drawback of this method is that concomitant polymorphs will not be distinguished until the crystals are dissolved again resulting in different equilibrium temperatures for the different polymorphic forms. Another drawback of the large number of crystals formed is the large change in supersaturation at the moment of nucleation and outgrowth. Both these drawbacks diminish the reliability of the polymorphic phase diagram considerably. We present a method that makes use of relatively small in-situ cells under well-controlled conditions. With these cells the metastable zone of each polymorphic form can be determined. By dissolving the nucleated crystals up to a single one, the equilibrium temperature of each form can be determined very precisely. Moreover, we can observe easily effects of chemical or physical changes in the growth conditions on the polymorphic form(s). Finally, it will be possible to selectively nucleate and grow one of these polymorphs.

Keywords: POLYMORPHISM, CRYSTAL GROWTH, NUCLEATION

Acta Cryst. (2002). A58 (Supplement), C144

\section{ELECTROSTATIC EFFECTS IN PROTEIN-UNFOLDING KINETICS: EXPERIMENT AND ENERGY CALCULATIONS}

A. Hernandez-Arana A. Rojo-Dominguez

Universidad Autonoma Metropolitana-Iztapalapa Quimica Apartado Postal 55-534 IZTAPALAPA D.F. 09340 MEXICO

The effect of ionic strength on protein folding/unfolding equilibrium and kinetics has frequently been used to probe the role of electrostatic interactions on such processes. In most cases, however, no attempts are made at discriminating between different mechanisms by which salts can affect conformational transitions. Besides exerting the well-known 'electrostatic screening', small ions in solution largely influence the stability of proteins because of preferential interactions with, or exclusion from, the macromolecular surface; these later interactions, which are salt-specific, are usually referred to as Hofmeister effects. We have determined rate constants for the unfolding reaction of chymopapain (a papain-related proteinase) under constant temperature and $\mathrm{pH}$, but at various concentrations of either $\mathrm{LiCl}$, $\mathrm{NaCl}$, or $\mathrm{Na}_{2} \mathrm{SO}_{4}$. By following the approach of Scholtz et al. (J. Am. Chem. Soc. 113, 5102), we have been able to separate specific Hofmeister effects, which predominate at high salt concentration, from a general dependence of the rate constant on ionic strength. This general effect thus represents an experimental benchmark for calculated electrostatic energy differences, between the native state and any putative transition-state conformation, as a function of ionic strength. The program DELPHI (an implementation for numerically solving the Poisson-Boltzmann equation) was used for shuch calculations; crystallographic coordinates of the protein molecule were used for the native state. 'Expanded' molecular conformations have been generated by several means. The structures of those conformations likely representing the transition-state ensemble are consistent with a rather small expansion of the native molecule.

Keywords: ELECTROSTATIC EFFECTS PROTEIN UNFOLDING TRANSITION STATES
KINETICS OF EXSOLUTION IN THE PENTLANDITEPYRRHOTITE ( $\left.\left(\mathrm{Fe}, \mathrm{Ni}_{\mathbf{9}}\right)_{\mathrm{S}_{8}}-\mathrm{Fe}_{(1-\mathrm{X})} \mathrm{S}\right)$ SYSTEM

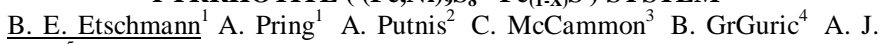
Studer ${ }^{5}$

${ }^{1}$ South Australian Museum Mineralogy Dept. North Terrace ADELAIDE 5000 AUSTRALIA ${ }^{2}$ Institute of Mineralogy, University of Muenster ${ }^{3}$ Geoinstitute, University of Bayreuth ${ }^{4}$ Nickel Division, Western Mining Corporation ${ }^{5}$ Neutron Scattering Group, Australian Nuclear Science and Technology Organisation

The kinetics of exsolution of pentlandite-pyrrhotite from the monosulphide solid solution (mss) were measured using powder neutron diffraction. Samples of the mss with compositions in the range Fe:Ni 9:1 to 7:3 were prepared using the standard evacuated silica tube method. The data collected from annealquench samples established that pentlandite exsolution is extremely rapid and that the solid solution in this system is non-quenchable. In situ heating/cooling diffraction experiments were performed with the pentlandite-pyrrhotite in an evacuated silica tube. A preliminary analysis of the data indicates that exsolution and phase transitions are fully reversible for synthetic samples, but not for naturally occurring samples. The proportion of nickel in the starting composition affects the exsolution characteristics. Long term anneal-quench experiments (up to 5 months) revealed that the textures observed in nature are due to diffusion processes continuing at temperatures less than or equal to 150 C. At higher temperatures geometric exsolution textures are produced.

Keywords: IRON NICKEL SULPHIDES, EXSOLUTION, KINETICS

\section{Acta Cryst. (2002). A58 (Supplement), C144 \\ IMAGING THE COMPETITION BETWEEN CHARGE-ORDERED AND CHARGE-DELOCALISED STATES IN $\mathrm{Pr}_{0.65}\left(\mathrm{Ca}_{\mathrm{y}} \mathrm{Sr}_{1-\mathrm{Y}}\right)_{0.35} \mathrm{MnO}_{3}$ \\ G.R. Blake ${ }^{1,2}$ P.G. Radaelli ${ }^{1}$ M. Gutmann ${ }^{1}$ D.N. Argyriou ${ }^{2}$ J.F. Mitchell ${ }^{2}$} ${ }^{1}$ Isis Rutherford Appleton Laboratory, Chilton DIDCOT OXFORDSHIRE OX11 0QX UK ${ }^{2}$ Materials Science Division, Argonne National Laboratory, Argonne, IL, 60439, U.S.A.

The role of local charge correlations and their competition with magnetic and electronic ground states is a major feature of the colossal magnetoresistive manganite perovskites. The double-exchange interaction competes with the electron-phonon interaction, giving rise to a metal to insulator transition at optimal doping. We have investigated the effect on this competition of tuning the A-site cation size while keeping the electronic doping level constant. We have carried out a detailed neutron powder diffraction study of the crystallographic and magnetic phase diagram of $\operatorname{Pr}_{0.65}\left(\mathrm{CaySr}_{1-\mathrm{y}}\right) 0.35 \mathrm{MnO}_{3}, \mathrm{y}=$ 0.6 to 0.8 , on the GEM diffractometer at ISIS. The high flux of GEM has allowed us to collect data at 28 temperatures for each of 5 compositions. A previous study of this system has suggested that a quasi-critical point may be present close to $y=0.75$, separating the ferromagnetic-metallic (FM) and charge-ordered insulating (COI) phases. However, we find no evidence to support this. We observe competition at the mesoscopic level between the FM and COI phases throughout the composition range studied, resulting in a coexistence of the two phases over large temperature ranges. The COI phase dominates on the Ca-rich side; in all compositions the FM phase fraction increases as the temperature is lowered, overcoming its suppression by the internal strain of a COI phase well established at higher temperatures. An additional feature of this system is a low-temperature spin reorientation in the COI phase.

\section{Keywords: COLOSSAL MAGNETORESISTANCE MANGANITES} PHASE DIAGRAM 